\title{
Analyser le jardin collectif urbain en géographie : une lecture du jardinage par les émotions
}

Camille Robert-Boeuf

\section{OpenEdition}

\section{Journals}

Édition électronique

URL : https://journals.openedition.org/cdg/1574

DOI : $10.4000 /$ cdg. 1574

ISSN : 2107-7266

Éditeur

UMR 245 - CESSMA

\section{Référence électronique}

Camille Robert-Boeuf, « Analyser le jardin collectif urbain en géographie : une lecture du jardinage par les émotions », Carnets de géographes [En ligne], 11 | 2018, mis en ligne le 15 septembre 2018, consulté le 20 mai 2021. URL : http://journals.openedition.org/cdg/1574 ; DOI : https://doi.org/ $10.4000 /$ cdg. 1574

Ce document a été généré automatiquement le 20 mai 2021.

\section{(c) (i) (9)}

La revue Carnets de géographes est mise à disposition selon les termes de la Licence Creative Commons Attribution - Pas d'Utilisation Commerciale - Pas de Modification 4.0 International. 


\title{
Analyser le jardin collectif urbain en géographie : une lecture du jardinage par les émotions
}

\author{
Camille Robert-Boeuf
}

\section{Introduction}

1 Le jardin est un objet de recherche traditionnel en sciences sociales, notamment étudié par les sociologues et les historiens (CABEDOCE \& PIERSON, 1996; DUBOST, 1997; WEBER, 1998). Pendant longtemps délaissé par les géographes, il redevient un sujet central des études géographiques au travers des problématiques sur le développement durable, l'agriculture urbaine et "l'habiter » (GALLIEN et al, 2012; MARLOIE \& BOUKHARAEVA, 2013 ; Mathieu, 2014 ; MENOZZI, 2014).

Mon travail de thèse aborde le jardin sous une de ses formes les plus anciennes, celle du jardin collectif ${ }^{1}$. Ces jardins sont des regroupements de parcelles, cultivées par des familles urbaines pour leur approvisionnement ou leur loisir, qui se sont développés tout au long du XXème siècle, en tissant des relations entre la ville et les périphéries rurales. Ces espaces présentent la particularité d'être des lieux multifonctionnels qui mêlent à la fois la fonction alimentaire, récréative, domestique ou encore la fonction sociale. Afin d'étudier cet objet de recherche, la plupart des travaux récents ont choisi de se concentrer sur une de ces fonctions: la fonction alimentaire (PLUVINAGE \& WEBER, 1992; POURIAS et alii, 2012; SCHEROMM, 2013). Néanmoins, toutes ces fonctions font du jardin collectif un espace fortement marqué par le sensible et le rapport à la terre. La difficulté pour le géographe est alors de comprendre un espace chargé en représentations et ayant une importante dimension émotionnelle. Dans cette optique, je désirerais ici réfléchir à comment analyser le jardin collectif urbain comme espace de l'ordre de l'émotion et quel positionnement de recherche cela implique.

3 Je construirai mon analyse en trois temps avec d'abord la compréhension du jardin comme un espace du quotidien, pour ensuite aborder sa dimension sensible comprise 
au travers de la géographie des émotions. Enfin j'évoquerai sa dimension collective qui impliquera un questionnement sur la position du chercheur sur son terrain puisque l'organisation sociale du jardin a induit une adaptation de mon positionnement par rapport aux enquêtés.

Cette réflexion se fonde sur mes travaux de thèse portant sur les jardins collectifs d'Ilede-France et de Kazan. Les enquêtes se sont concentrées sur quatre regroupements de parcelles en Ile-de-France et autant à Kazan, des recherches préliminaires ayant été effectuées au cours de mes deux Masters sur plusieurs autres sites de ces mêmes régions. Je m'appuierai principalement sur la pratique du terrain dans les jardins, le corpus d'enquêtés se composant avant tout de jardiniers : vingt entretiens de type récits de vie ainsi que plusieurs dizaines d'entretiens et de discussions informelles ont été effectués et 200 questionnaires ont été récoltés. Toutefois ces recherches m'ont également conduit à m'entretenir avec des responsables associatifs, des acteurs publics ayant en charge la gestion de jardins, des juristes et des chercheurs spécialisés sur les questions foncières et d'agriculture urbaine. Aux questionnaires et entretiens, souvent utilisés en géographie, se sont greffées d'autres techniques, empruntées à la sociologie ou à l'anthropologie, que nous allons explicités tout au long de l'article.

\section{Concevoir le jardin comme territoire du quotidien}

Les jardins collectifs en Ile-de-France et dans la région de Kazan sont des espaces qui se structurent en périphérie de la ville, dans des délaissés urbains ou sur du foncier agricole peu exploitable par l'agriculture intensive (terres près des axes de communication ou à la topographie escarpée, etc.). Aujourd'hui gérés par des associations, ils ont toujours été très encadrés par l'Etat qui a produit plusieurs séries de lois afin d'orienter et de mettre en avant une fonction plutôt qu'une autre. Sur les deux terrains de recherche, les jardins sont depuis plusieurs années confrontés à l'urbanisation massive de l'espace périurbain et se sont transformés pour répondre aux besoins des citadins. Ayant eu la même fonction d'origine, répondre aux besoins alimentaires de la ville, ils se sont massivement développés dans l'entre-deux guerres puis pendant la seconde guerre mondiale. Entre la fin du XXème siècle et le début des années 2000 , la fonction de loisir est devenue de plus en plus importante, ces espaces acquérant de nouveaux rôles: reflet d'une nouvelle périurbanisation des quartiers périphériques ou espaces favorisant un contact à la nature à Kazan; lieu de l'agriculture urbaine propice à la protection de l'environnement et à la création du lien social en Ile-de-France. Dans ce contexte, la mise en regard de ces deux terrains permet d'observer, sur le temps long, leur adaptabilité et l'évolution des différentes fonctions tour à tour mises en valeur soit par les jardiniers soit par les acteurs publics.

Depuis leur origine, ces jardins gardent pourtant une fonction primordiale: la production agricole. Ce sont des espaces où les urbains travaillent la terre de manière quotidienne, le jardinier entretient sa parcelle tous les jours pendant la saison estivale et revient régulièrement en hiver soit pour récolter quelques légumes et travailler le sol en Ile-de-France, soit pour vérifier le bon état des abris et maisonnettes à Kazan ${ }^{2}$. Il retourne la terre à l'automne et la prépare pour l'hiver en y mettant du fumier et des déchets verts. Au début du printemps il prépare ses semis, calcule l'organisation de sa parcelle qui change toutes les années avec la rotation des cultures et prévoit la structuration du potager et les différentes associations de légumes pour optimiser les 
récoltes (certaines plantes en protègent d'autres, comme l'oignon qui repousse les insectes). L'acquisition de graines se fait en amont ou dès l'été précédent si le jardinier les produit lui-même, ce qui arrive quelques fois quand il en a l'expérience ou pour certaines variétés, comme la tomate où la récupération des graines n'est pas difficile. Ensuite les semis sont plantés et la parcelle doit être arrosée régulièrement et les mauvaises herbes arrachées. Les premières récoltes se font en été et au fur et à mesure de celles-ci, les jardiniers peuvent replanter pour avoir une deuxième voire une troisième récolte dans la saison. Tout au long de l'été, les légumes sont soit consommés soit conservés pour l'hiver, certaines personnes pouvant se nourrir en grande partie à partir de leur jardin. Pour la partie construite de la parcelle, l'abri ou la maisonnette sont régulièrement, soit agrandis ${ }^{3}$, soit repeints ou lasurés. Cette partie de la parcelle reflète l'aspect véritablement domestique du jardin : on y met une petite table pour recevoir ses amis et on l'aménage en rapportant des objets de chez soi ou récupérés par exemple. Le jardinage est une activité qui exige un dur labeur grâce auquel « on se vide la tête ", " on est au calme ", comme le dit par exemple une jardinière de Nanterre : "[Le jardin] c'est un supplément d'âme... Vraiment. J'avais besoin de verdure. Moi je ne suis pas d'ici, hein, je suis une immigrée. Donc j'avais besoin d'avoir les mains dans la terre, j'avais besoin d'un espace pour respirer ». Plus qu'un espace d'activité agricole, c'est un espace domestique qui se révèle être une véritable extension de la résidence principale voire de l'individu faisant appel aux souvenirs nostalgiques de l'enfance, comme le montre cet extrait d'entretien avec une fille de jardinière russe: "en fait nous vivions à la datcha avec mes amies soit ici [dans sa datcha] soit chez Aliona [...]. On achetait rarement à manger, on se servait de ce qui poussait dans le jardin. La datcha ce n'est pas un simple endroit en périphérie de la ville, c'est un lieu que l'on chérit, tout le monde aime la datcha, on peut s'y reposer, faire des fêtes [...] ». Le jardin apparaît alors comme un espace mouvant puisque toujours en évolution pour répondre aux besoins de son occupant mais aussi intimement lié à l'histoire individuelle de ce dernier et producteur d'émotions. La parcelle se transforme au fur et à mesure de la vie du jardinier oscillant entre production intensive lorsque celui-ci a besoin de se nourrir (en période de crise économique, après une baisse de ses revenus ou lorsque sa famille s'agrandit) et espace de loisir, quand le besoin alimentaire est plus faible.

7 C'est, de plus, un espace aux mutations subtiles puisque les pratiques agricoles utilisées - la rotation des cultures et l'organisation des plants en allées géométriques notamment - font référence aux techniques agricoles des maraîchers du XIXème siècle, donnant l'impression d'un espace immuable. Pour donner à voir les évolutions du jardin, qui peuvent être de l'ordre de l'arrangement des allées au sein de la parcelle et se faire à l'échelle micro-locale, j'ai décidé d'utiliser la méthode du récit de vie. Au cours des entretiens préliminaires, j'ai remarqué que les enquêtés faisaient beaucoup référence à différentes étapes de leur vie, la naissance de leur premier enfant revenant régulièrement. Pour approfondir ce lien entre jardin et vie personnelle, il m'a paru intéressant de rallonger mes entretiens en ouvrant plus largement la grille de questions sur les activités de la vie quotidienne, les pratiques de consommation alimentaire et de loisirs, et sur la vie de l'enquêté avant l'acquisition du jardin, c'est-àdire son enfance et son parcours professionnel. Ces entretiens se faisaient en plusieurs parties, soit dans les parcelles, soit chez la personne enquêtée. Lors de la première rencontre, les questions étaient plus orientées sur les pratiques concrètes effectuées dans la parcelle et, au fur et à mesure des visites, l'entretien pouvait aborder des questions de l'ordre de la vie privée et se transformer en véritable discussion. Ces allers 
et retours auprès des mêmes jardiniers, devenus souvent des enquêtés-ressources au sein du regroupement de parcelles, permettaient, en même temps que de saisir un discours plus personnel, de capter le quotidien des jardiniers à plusieurs étapes du jardinage et de percevoir, moi-même, les évolutions de la parcelle sur plusieurs mois ou d'une année sur l'autre. Pour rendre visible ces changements, j'ai pris des photographies lors des différentes visites, conservant plusieurs clichés d'une même parcelle. Ces photographies, considérées comme support à l'entretien, ont servi d'illustrations pour les discours enregistrés tout en approfondissant le travail d'analyse à l'échelle micro-locale et suivant les différentes saisons. Cette manière d'aborder le discours au travers du récit de vie a favorisé l'analyse de l'expérience humaine "sur le vif » et de son «épaisseur subjective » (MOHIA, 2000, p. 276) et la reconstruction de la «trajectoire biographique» de l'individu. Cela a permis de voir comment le jardin s'inscrit dans son quotidien en faisant apparaitre son épaisseur historique (MORANGE \& SCHMOLL, 2016).

\section{Saisir le sensible dans les jardins : une géographie des émotions}

8 Le jardinage est une activité qui appelle l'émotion, comme nous l'avons vu avec la référence à la nostalgie de l'enfance, mais aussi parce qu'elle demande une utilisation accrue du sensible, c'est-à-dire des cinq sens.

La pratique du jardinage exige tout d'abord la mobilisation du touché ; le contact à la terre est un élément important souligné dans plusieurs de mes entretiens avec des expressions comme " avoir les mains dans la terre » ou encore " avoir la main verte » qui reviennent régulièrement. Ensuite, le cadre du jardin appelle l'ouïe et l'odorat avec un attachement des jardiniers à l'observation de la nature et de son évolution. La plupart des jardiniers notent les changements de saisons, comme en témoigne cet extrait de discussion avec un jardinier de Melun : « Déjà ça change, l'été est fini, on voit que les arbres changent. [...] Et puis globalement la nature est pas la même qu'avant, ce ne sont pas les mêmes plantes qui poussent, on voit l'influence du changement climatique. ». Mais les sens les plus importants sont le goût et la vue. Les jardiniers apprécient leurs récoltes, reconnaissent les différentes variétés, apprennent et enseignent la dimension qualitative des produits et donc leur goût. C'est tout particulièrement le cas pour les tomates dont le goût est jugé incomparable par rapport à celles trouvées dans le commerce. Les légumes sont avant tout plantés pour leur saveur et la grande majorité des enquêtés préfèrent la variété des légumes à la quantité, la règle invoquée étant de faire « un petit peu de tout » tant en Russie qu'en France. On y retrouve généralement les mêmes légumes, essentiellement tomates, choux, salades, concombres, poivrons, betteraves, pommes de terre et oignons. Plus largement, le goût renvoie au repas que les jardiniers partagent avec leurs invités, il légitime leur valeur au travers de celle des récoltes : le bon jardinier est celui qui peut produire de bons légumes et in fine cuisiner de bons plats. Enfin, la vue renforce la valorisation des récoltes puisqu'il n'est pas rare que les enquêtés photographient leurs plus beaux légumes ou leurs plus belles fleurs pour les montrer à leurs visiteurs et en conserver le souvenir. La mobilisation de l'ensemble des cinq sens pour le jardin contribue à en faire des espaces du sensible et crée l'impression d'un monde à part, un monde idyllique grâce auquel on peut quitter sa résidence principale pour un meilleur 
cadre de vie, ainsi une jardinière de Kazan dit : «Ce qui me plait le plus c'est la nature, être proche de la nature, la verdure [...]. Le matin, quand le soleil commence à éclairer le jardin, je peux regarder les fleurs et pleurer parce que cela me fait du bien, parce que c'est beau, parce qu'ici règne le silence. ». Plus que de la simple nostalgie d'un passé lointain, le jardin apporte de la satisfaction et de la fierté d'avoir de bonnes récoltes et de la joie d'accéder à une meilleure qualité de vie.

10 Afin de faire apparaitre cette dimension sensible du jardin et des émotions qu'il apporte, je me suis rendue compte que le récit de vie, même couplé à la photographie, ne suffisait pas, notamment pour percevoir la "dimension spatiale des émotions " (GUINARD \& TRATNJEK, 2016). J'ai alors décidé de recourir au parcours commenté, méthode au cours de laquelle l'enquêteur arpente au côté de l'enquêté l'espace étudié, pendant que ce dernier décrit le lieu visité. Cette technique pose comme hypothèses que « la perception se déploie en fonction d'un milieu bien plus que dans un milieu » et que « le sensible est un formidable embrayeur de parole » (DELAS, 2015, pp. 55-56). Ma pratique du parcours commenté s'est faite, sous plusieurs formes, du parcours descriptif de l'espace jardiné à la déambulation aléatoire. Mais elle a été systématique au sein des parcelles visitées et il a été invariablement demandé à chaque enquêté de décrire son jardin. La demande de visite du jardin n'a d'ailleurs pas toujours été nécessaire, certains enquêtés m'invitant naturellement à parcourir leur parcelle pour montrer leur travail. Cette pratique au cœur des jardins a permis de délier une parole difficile pour des personnes qui n'ont pas l'habitude de se raconter. Parfois, elle consistait même en un rituel qu'effectuent les jardiniers quand ils reçoivent des invités: une sorte d'initiation au jardin pour qu'il devienne un espace familier, le visiteur passant d'étranger à initié. J'ai ensuite utilisé le parcours commenté à une plus petite échelle avec des présidents ${ }^{4}$ de jardins qui nous ont présenté l'ensemble du jardin collectif. Dans ces cas-là, le parcours a inclus le tour des différentes allées et des infrastructures communes. Dans une approche plus réflexive, cette technique d'enquête a révélé les relations que tissaient les présidents avec les jardiniers croisés, tout en mettant au jour les jardins montrés en exemple et ceux critiqués. L'exemple des jardins familiaux de Melun peut être sur ce point éclairant: en faisant le tour des parcelles avec le président de l'association j'ai été invité à admirer les jardins considérés comme les mieux entretenus. La rencontre avec certains jardiniers considérés comme problématiques pour le président a pu amener notre conversation sur des problèmes spécifiques, notamment la question des parcelles peu entretenues ou des problèmes de voisinages ou avec les services de la mairie. Là encore la photographie m'a fourni un support efficace, les jardins montrés en exemple ou critiqués étant systématiquement photographiés. Les matériaux récoltés ont été comparés en fonction des différents sites visités et j'ai pu faire ressortir des normes esthétiques qui se retrouvent dans tous les jardins, tant sur le terrain russe que sur le terrain français, comme par exemple l'exigence d'un sol nettoyé de toute mauvaise herbe, des rangs de légumes bien alignés, d'une grande variété de légumes, de fruits et de fleurs (voir figure 1). 
FIgURE 1 : DEUX PARCELLES DES JARDINS FAMILIAUX DES CARMES (MELUN)
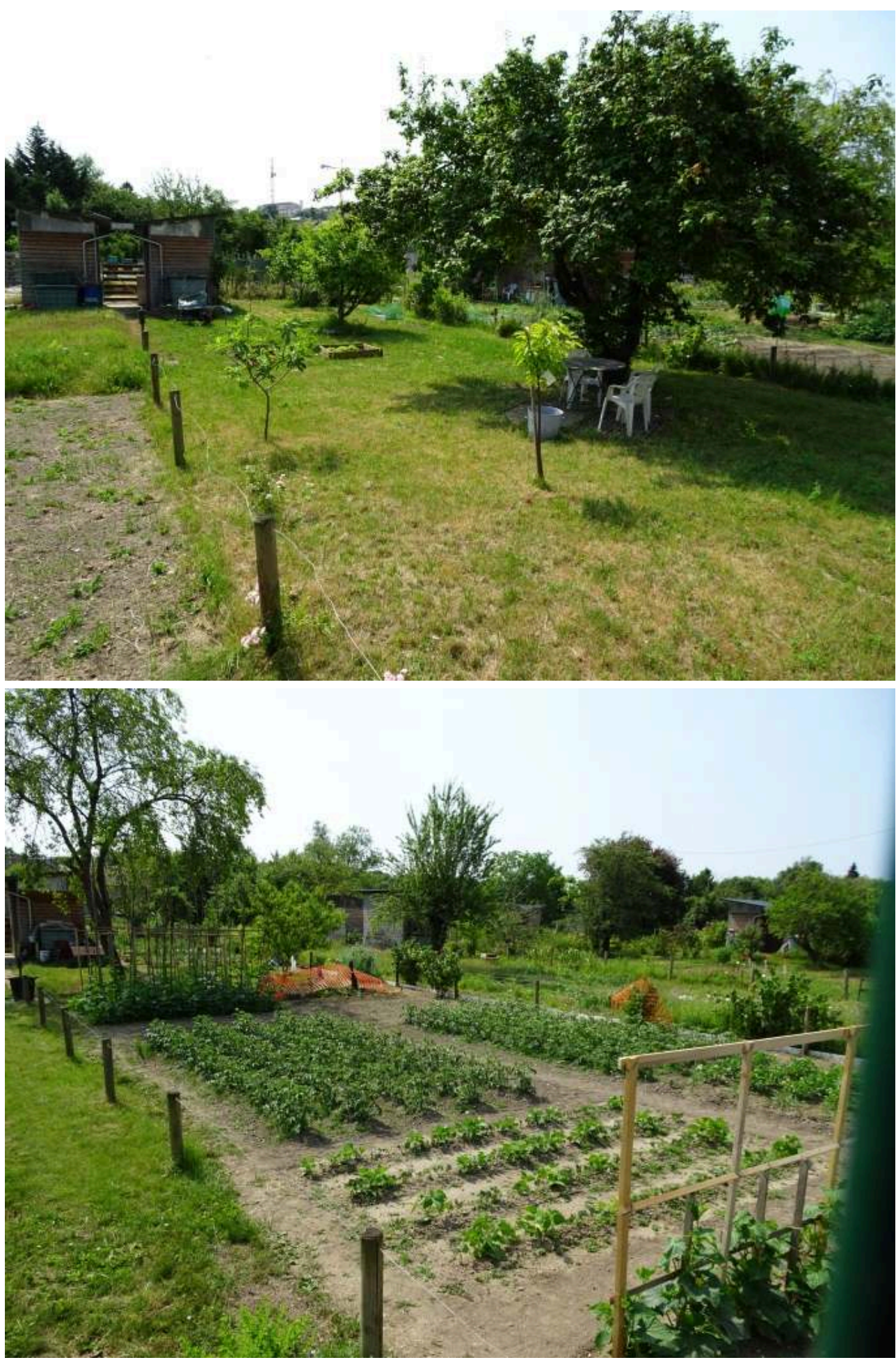

Source/crédit : Camille Robert-Boeuf, 2017

Ces deux photographies, prises lors du parcours commenté effectué aux jardins de Melun, montrent deux exemples de parcelles diamétralement opposées : celle de gauche présente un jardin peu entretenu c'est-à-dire avec très peu de plantations, la parcelle n'est pas cultivée à l'exception d'un bac au centre, son occupant est alors jugé mauvais jardinier ; celle de droite affiche un jardin jugé exemplaire, le sol y est nettoyé de toute herbe, les rangées de légumes bien alignées et la parcelle majoritairement occupée par les plantations. 
11 Le jardin est un espace qui externalise le sensible dans sa dimension globale en aiguisant chez le jardinier ses cinq sens simultanément, déployant un registre important d'émotions (la joie, la satisfaction, la sérénité, la nostalgie, la tristesse, etc...). Ces émotions sont valorisées par les enquêtés et sont bien souvent la raison qui les pousse à jardiner. Ce rapport entre émotion et jardin est tel qu'il arrive que des jardiniers projettent leurs sentiments sur leur parcelle, cette dernière devenant laide et mal entretenue à leurs yeux quand ils sont malheureux ou connaissent des difficultés notamment. La recherche d'émotions et de rapports concrets à l'espace est donc l'un des moteurs du jardinage. Grâce au parcours commenté, j'ai pu souligner ce lien entre jardin et sensible qui inscrit cet espace de plein pied dans la géographie des émotions.

\section{Le jardin comme collectif : quel positionnement du chercheur au sein d'un réseau de sociabilités?}

12 Malgré leur organisation en parcelles individuelles, les jardins sont des lieux de sociabilités fortes, où les relations interpersonnelles se construisent à plusieurs échelles au travers des repas, des activités agricoles collectives, des dons, et des échanges de conseils et de récoltes.

13 Le premier cercle de sociabilités se construit à l'échelle familiale, au travers du jardinage effectué dans les parcelles puisque l'apprentissage se fait en grande partie auprès des parents ou des grands-parents. Les jardiniers établissent ensuite des liens forts à l'échelle des parcelles voisines, par regroupements de quatre ou cinq parcelles. Les enquêtés connaissent très bien leurs voisins et échangent régulièrement conseils et récoltes, s'entraident pour les travaux difficiles ou lorsque l'un d'entre eux est malade. Cela aboutit parfois même à la suppression des clôtures entre parcelles voisines. A l'échelle du jardin collectif, les sociabilités se structurent autour des membres du Conseil d'administration et surtout du président de l'association. Celui-ci joue un rôle central dans la circulation des informations entre jardiniers et entre les jardins et les acteurs extérieurs, principalement les services de la ville et les associations de jardins régionales et nationales 5 . En effet, le président est la cheville ouvrière de l'association, il veille à ce que le règlement soit appliqué, résout les conflits et répond aux requêtes des autres membres.

14 Les jardins forment un groupe d'interrelations soudées qui passent par une expérience collective du jardinage et des émotions provoquées par cette activité. Ils définissent un "nous » (ensemble des membres de l'association) par rapport à un " eux " (personnes extérieures au jardin). Cela s'observe concrètement dans la manière dont les allers et venues dans le jardin sont souvent surveillées (par le président ou par des jardiniers proches des entrées) voire contrôlées. Cette construction sociale des jardins m'a obligé à adapter ma méthodologie : le jardin est en apparence un espace ouvert puisque je pouvais y rentrer librement toute la journée. Cependant, l'introduction par un membre de l'association était nécessaire pour pouvoir discuter librement avec les jardiniers et ne pas être considérée comme une hypothétique voleuse ou une représentante des autorités publiques. Afin d'être acceptée par le collectif, il a fallu me présenter et prouver ma légitimité en répondant parfois à des devinettes et questions des jardiniers qui testaient mes compétences en jardinage. Sur le terrain russe, j'ai dû justifier ma présence en tant que française, pas toujours acceptée ou comprise, en montrant ma 
connaissance de la région et de la culture du pays. En France comme en Russie, il m'était par ailleurs fréquemment posé des questions sur mes origines et ma pratique personnelle du jardinage de manière, sans doute, à savoir si je pouvais être considérée comme une jardinière à part entière. Pour aider à cette intégration, j'ai utilisé l'observation et notamment l'observation participante, validant ainsi aux yeux des jardiniers mon savoir-faire. L'observation a permis de me faire accepter et de me glisser, le temps d'un moment, «dans la peau du jardinier » et de m'approprier ses expériences sensibles. Le temps du repas, qui va de sa préparation à la fin du repas, a été le point central de ma pratique de l'observation. Mon intégration à la préparation $\mathrm{du}$ repas pouvait signifier que j'étais devenue un membre, même éphémère, de l'association, formant des liens interpersonnels avec les jardiniers au-delà de la réalisation d'entretiens. De plus, partager un repas issu de sa propre récolte signifie, pour le jardinier, partager une partie de soi, de son travail et par ma participation à ces repas je reconnaissais son travail. Devoir se faire accepter au sein de la communauté jardinière m'a en conséquence demandé de multiplier mes visites afin d'être perçue comme une personne connue et habituelle.

La nécessité de tisser des liens avec les enquêtés et la pratique de l'observation m'ont enfin permis de créer un recul critique entre les discours et les pratiques réelles. Si c'est vrai pour toute recherche en science sociale, c'est particulièrement nécessaire lors d'enquêtes dans les jardins collectifs car, ceux-ci étant de plus en plus étudiés et ayant une dimension symbolique et normative forte, les discours formatés ou du moins conventionnels sont nombreux. Ces discours sont, d'une part, influencés par les normes instaurées au sein des associations qui définissent un ensemble de bonnes et de mauvaises pratiques. De cette manière, peu d'enquêtés avouent utiliser des pesticides même en Russie où cela n'est pas interdit car c'est mal vu par l'ensemble de la communauté jardinière. D'autre part, la présence de plusieurs chercheurs en amont de mon enquête sur les terrains choisis ont pu également impacter le discours des jardiniers. M. Marloie et L. Boukharaeva, par exemple, ont travaillé sur les jardins collectifs de Kazan dans les années 2000 et ont permis à des membres de l'Association des Jardiniers de Kazan et de la Fédération Nationale des Jardins Familiaux et Collectifs de se rencontrer, en invitant des jardiniers français à visiter des jardins russes et inversement (MARLOIE \& BOUKHARAEVA, 2013). Ces relations, construites grâce aux chercheurs, ont créé la circulation d'argumentaires et de représentations spécifiques sur les jardins : les membres de l'association russe ont renforcé leurs discours sur leur rôle écologique dans la ville en utilisant un vocabulaire renvoyant par exemple à la permaculture jusque-là peu utilisé ${ }^{6}$; les membres des associations françaises ont, quant à eux, pu utiliser l'exemple des jardins russes pour montrer la dimension internationale du jardinage afin d'affirmer la nécessité de leur existence face à d'autres acteurs. Le chercheur n'est plus quelqu'un de neutre ou d'extérieur, «sa présence sur un temps long fait de lui un personnage ayant un rôle interne. » (ALTHABE et HERNANDEZ, 2004). Il devient un outil pour les enquêtés, ce qui, dans la situation particulière des deux terrains choisis, a exigé d'accorder une attention toute particulière à la prise en compte des écarts entre discours et pratiques. Cela a permis de démêler les prises de position des pratiques réelles mais aussi de dégager plusieurs modèles de jardins (jardin agricole, domestique, naturel, de loisir, etc...) plus ou moins revendiqués en fonction des protagonistes considérés ; si les associations affirment cet espace comme un lieu de promotion écologique et de sociabilités, les jardiniers le revendiquent avant tout comme un espace domestique. Ainsi, l'articulation de plusieurs méthodes d'enquêtes a 
favorisé une meilleure compréhension des relations entre acteurs et la prise en compte des effets de catégorisation qui se cristallisent autour du jardinage, tant dans les discours des enquêtés que chez les chercheurs.

\section{Conclusion}

Les jardins collectifs, tels que je les ai définis, sont des lieux mouvants qui évoluent tant en fonction des saisons que sur le temps long et marquent le quotidien des jardiniers impliquant des pratiques, des représentations et des sociabilités qui se structurent autour d'un emboîtement complexe d'échelles. C'est de ce fait un espace de l'ordre de l'émotion, où se crée une dimension sensible et collective du jardinage qui montre la manière dont les individus s'ancrent dans le territoire et l'impactent grâce à la pratique et les échanges agricoles.

Faire émerger l'espace sensible du jardin a exigé une méthodologie invoquant l'articulation de techniques d'enquêtes qualitatives employées en géographie mais également en sociologie ou en anthropologie. Aux entretiens et questionnaires il a fallu ajouter l'entretien de type récit de vie, l'observation et le parcours commenté, la photographie servant à renforcer et illustrer les résultats obtenus. J'ai alterné les différentes méthodes de manière à ce qu'elles trouvent des échos les unes avec les autres, les parcours commentés servant de point de départ aux récits de vie et facilitant l'observation et inversement les entretiens et l'observation permettant la réalisation de parcours commentés. La pratique du jardin s'est construite sur une suite d'allers et retours multiples pour percevoir sa saisonnalité et pour mieux m'intégrer au sein de la communauté des jardiniers. Etudier le jardin signifie s'inscrire dans la méthodologie de la géographie des émotions et affirmer le jardinage comme pratique émotionnelle tout en impliquant le chercheur au plus près de son terrain, celui-ci devant dépasser le couple classique enquêté-enquêteur pour mettre au jour des évolutions micro-locales et des effets de catégorisation.

\section{BIBLIOGRAPHIE}

Althabe G., Hernandez V. A., 2004, « Implication et réflexivité en anthropologie », Journal des Anthropologues, 98-99, [En ligne].

Cabedoce B., Pierson P., 1996, Cent ans d'histoire des jardins ouvriers : 1896-1996 La Ligue Française du Coin de Terre et du Foyer, Grâne, Editions Créaphis.

Delas J., 2015, « Cheminer « en aveugle » dans des lieux inconnus : perspectives réflexives sur la situation d'enquête », Spécificités, 2, 54-59.

Dubost F., 1997, Les jardins ordinaires, Paris, l'Harmattan.

Gallien M, Boitard M, Delfosse C., 2012, « Renouveau des jardins potagers entre milieux urbain et rural ? », Pour, 3, 321-332. 
Guinard P., Tratnjek B., 2016, "Géographies, géographes et émotions », Carnets de Géographes, 9, [En ligne].

Marloie M., Boukharaeva L., 2013, L'utilisation des sols urbains et périurbains pour le développement humain durable des villes : une alternative au modèle pavillonnaire, rapport fait dans le cadre $\mathrm{du}$ programme GESSOL, Paris, Fondation Maison des Sciences de l'Homme.

Mathieu N., 2014, « Jardin de ville, jardin de campagne, quel rôle dans l'émergence d'un mode d'habiter durable? », in : Menozzi M-J. (sous la direction de), Les jardins dans la ville entre nature et culture, Rennes, Presses universitaires de Rennes.

Menozzi M-J., 2014, Les jardins dans la ville entre nature et culture, Presses universitaires de Rennes, Rennes.

Mohia N., 2000, « Le récit de vie : une approche anthropologique heuristique ? », L'Autre, 2, 259-279.

Morange M., Schmoll C., 2016, Les outils qualitatifs en géographie : méthodes et applications, Armand Colin, Malakoff.

Pluvinage M., Weber F., 1992, Les jardins populaires : pratiques culturales, usages de l'espace, enjeux culturels : éléments d'histoire et d'ethnographie : rapport de recherche pour la Mission du Patrimoine ethnologique du Ministère de la Culture, Paris, Ministère de la culture.

Pourias J., Daniel, A-C., Aubry C., 2012, « La fonction alimentaire des jardins associatifs urbains en question », Pour, 3, 333-347.

Scheromm P., 2013, « Les jardins collectifs, entre nature et agriculture », Métropolitiques, [En ligne].

Weber F., 1998, L'honneur des jardiniers, les potagers dans la France du XXe siècle, Paris, Belin.

\section{NOTES}

1. Les jardins collectifs, assimilés aux jardins familiaux ou ouvriers en France et aux datchas en Russie, se distinguent des jardins partagés, individuels, pédagogique, d'insertion, etc...

2. A cause du climat continental et du mauvais état des routes, les jardiniers russes ne reviennent pas fréquemment dans les jardins collectifs en hiver.

3. Notamment en Russie puisqu'aucune règlementation ne régule leurs dimensions depuis 1985, certains en profitant pour édifier de véritables résidences estivales.

4. Les jardins étant des associations de type loi 1901, ils possèdent tous un Conseil d'administration avec un président élu pour deux ou trois ans.

5. En Ile-de-France, ces associations sont au nombre de deux : la Fédération Nationale des Jardins Familiaux et Collectifs et Jardinot. A Kazan, une seule association, l'Association des Jardiniers du Tatarstan, représente les jardiniers.

6. Cela ne veut pas dire que le rôle écologique et les pratiques respectueuses de l'environnement n'existaient pas avant, bien au contraire, mais les discours ne les mettaient pas toujours en avant. 


\section{RÉSUMÉS}

Les jardins collectifs sont des espaces multifonctionnels et profondément évolutifs qui s'inscrivent dans le quotidien des urbains et favorisent l'externalisation du sensible. Ces spécificités appellent une méthodologie particulière, mêlant plusieurs techniques d'enquêtes qualitatives que sont le récit de vie, le parcours commenté et l'observation. Au travers de deux terrains, les jardins franciliens et ceux de la région de Kazan (Russie), je souhaiterais réfléchir à la manière dont le géographe peut enquêter un espace relevant de la géographie des émotions et questionner ses relations au terrain.

Collective gardens are multifunctional and deeply evolving spaces, they are part of the city dweller's daily life and promote their sensitivity. These specificities call for a methodology, combining several techniques of qualitative surveys. Through our two fields, the gardens of the Ile-de-France and those of the Kazan region, we aim to reflect on how the geographer can investigate a space linked to the geography of emotions. We also would like to interrogate the relationship of the researcher with this typical field.

INDEX

Thèmes : Carnets de terrain

Keywords : collective gardens, methodology, geography of emotion, fieldwork.

Mots-clés : jardins collectifs, méthodologie, géographie de l'émotion, terrain

\section{AUTEUR}

\section{CAMILLE ROBERT-BOEUF}

UMR LAVUE

Université Paris Nanterre

kamrb@hotmail.fr 